
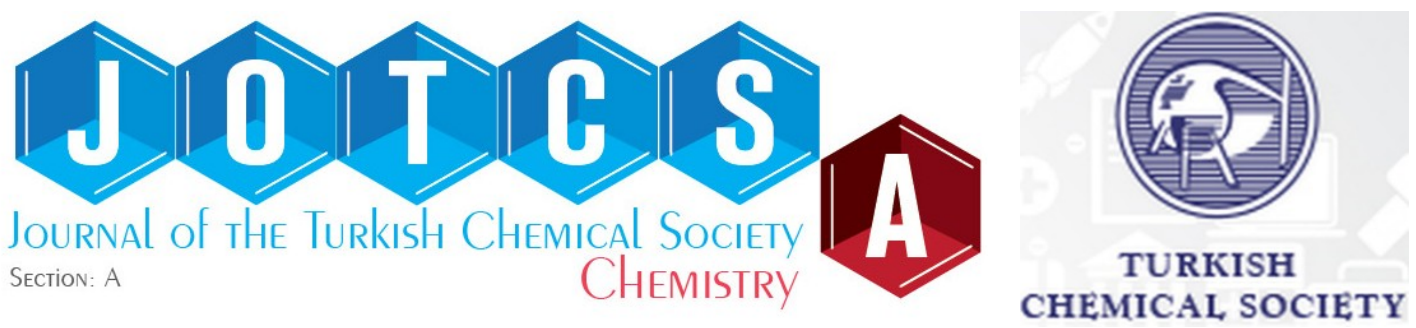

\title{
Effect of Inorganic Components of Fire Foaming Agents on the Aquatic Environment
}

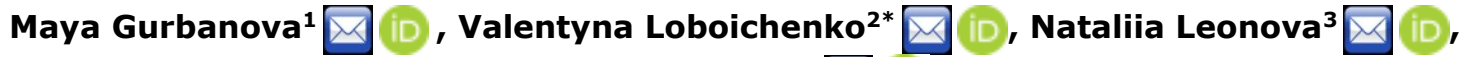 \\ Victor Strelets 4
}

\author{
${ }^{1}$ Academy of Ministry of Emergency Situations of the Azerbaijan Republic, AZ1089, Baku, Azerbaijan \\ Republic \\ ${ }^{2}$ Department of Occupational, Technogenic and Environmental Safety, National University of Civil Defense \\ of Ukraine, 61023, Kharkiv, Ukraine \\ ${ }^{3}$ Chemical Metrology Department, Kharkiv National University Named by V.N. Karazin, 61022, Kharkiv, \\ Ukraine \\ ${ }^{4}$ Scientific Department on Problems of Civil Protection and Technogenic and Ecological Safety, National \\ University of Civil Defence of Ukraine, 61023, Kharkiv, Ukraine
}

\begin{tabular}{|l||}
\hline Abstract: Impact on the aquatic medium of the number of inorganic additives that are part of the \\
foaming agents for firefighting is investigated in paper. The influence of the most widespread inorganic \\
components on aquatic organisms was analyzed. Significant variability of data was noted. It is proved \\
that the magnesium and sodium chlorides are the safest for the environment and the most dangerous \\
ones are aluminum compounds and sulfamic acid. Inorganic additives based on aluminum, sulfamic \\
acid, and sodium bicarbonate are the most dangerous for aquatic living organisms, in the short and \\
long term, and the safest compounds are magnesium and sodium chlorides.
\end{tabular}

Keywords: Fire foaming agents, inorganic additives, aquatic environment.

Submitted: August 27, 2020. Accepted: October 06, 2020.

Cite this: Gurbanova M, Loboichenko V, Leonova N, Strelets V. Effect of Inorganic Components of Fire Foaming Agents on the Aquatic Environment. JOTCSA. 2020;7(3):833-44.

DOI: https://doi.org/10.18596/jotcsa.785723.

*Corresponding author. E-mail: vloboichm@gmail.com, Tel: +38 0507509864.

\section{INTRODUCTION}

Today's environment is often subject to anthropogenic impact. Apart from the work of industrial enterprises, agricultural facilities, housing and communal sector, the influence of various types combustion that turn into fires is also negative. Fires often acquire catastrophic scale affecting individual technogenic objects (1) and the planet as a whole $(2,3)$ despite the preventive measures $(4,5,6)$.

Today, one of the most effective means for localizing and extinguishing fires of various types, including oil products, is foam. During the fire both firemen (7) and environmental objects are exposed to dangerous thermal effects. At the same time, the environment is negatively affected both by the fire itself (8) and the ingress of combustion products and components of fire extinguishing mixtures into the air, water, and soil $(9,10)$. As the latter ones, apart from foams (11), the water (12) and fire extinguishing powders (13) often act.

According to the composition, foaming agents are divided into synthetic, protein, fluorosynthetic, and fluoroprotein ones (14). They are a mixture of organic and inorganic compounds of natural or artificial origin. In addition to identifying $(15,16)$ and evaluating the content of these compounds 
and their decomposition products in the environment using various laboratory (17) and express physical and chemical methods of analysis $(18,19)$, it is important to study their ecological properties.

Detergents (20), alkyl sulfonates (21), fatty acids (22), natural compounds (23), and fluorinated derivatives (24) are used as the main active ingredients.

The influence of the main substances, which are present in the foaming agents, on the environment, mainly, aquatic environment, has been sufficiently studied $(25,26)$. As ecological and ecotoxicological characteristics, experimental or calculated bioindication parameters appear in this case.

On the contrary, it should be noted that in addition to the main substance, various additives are also included in the foams, which affect on the properties of the foams such as multiplicity, viscosity, stability, frost resistance, etc. These additives are organic (alcohols, acids, and their salts) or inorganic compounds. When extinguishing the fires, these additives also enter the environment and have a negative impact on it.

The policy of developers and manufacturers to replace the precise composition of the foaming agent with a trade name, brand or generic name, including the Safety Data Sheets (27), greatly complicates the assessment of the environmental characteristics and environmental impact of individual components of the foaming agents. The information about the environmental impact of individual components of the foaming agents can be useful while developing new, more environmentally friendly compounds of the foaming agents. It will also allow potential buyers to make more environmentally conscious choices when purchasing these products.

The aim of this paper is a comparative study of the impact on the environment, in particular, the aquatic environment of the individual inorganic components of the foaming agents.

\section{MATERIAL AND METHODS}

The well-known analytical methods of processing the data are used in the paper by applying the information about chemicals presented in the literature and on the website of European Chemicals Agency (28). As the parameters of research, the values PNEC - predicted no effect concentration, LC - Lethal concentration, NOEC no observed effect concentration, LOEC - lowest observed effect concentration, ECx the effect concentration associated with $\mathrm{x} \%$ response $(27$, 29) are selected.

\section{RESULTS AND DISCUSSION}

In the paper, the approach proposed in $(13,30)$ was used. The essence of the proposed assessment is to study the environmental characteristics of inorganic salts which are used to improve the extinguishing properties of foaming agents. Their composition can vary from a few thousandths to tens of percent of the total mass of the substance $(0.005-40 \%)$.

The best known (31-40) additives are compounds such as magnesium chloride and its natural analogue, bischofite $\left(\mathrm{MgCl}_{2}\right)$, basic aluminum chloride $\left(\mathrm{Al}_{2}(\mathrm{OH})_{5} \mathrm{Cl}\right)$, sulfamic acid $\left(\mathrm{NH}_{2} \mathrm{SO}_{3} \mathrm{H}\right)$, sodium bicarbonate $\left(\mathrm{NaHCO}_{3}\right)$, calcium chloride $\left(\mathrm{CaCl}_{2}\right)$, sodium chloride $(\mathrm{NaCl})$, sodium carbonate $\left(\mathrm{Na}_{2} \mathrm{CO}_{3}\right)$, ammonium sulfate $\left(\left(\mathrm{NH}_{4}\right)_{2} \mathrm{SO}_{4}\right)$, aluminum sulfate $\left(\mathrm{Al}_{2}\left(\mathrm{SO}_{4}\right)_{3}\right)$, sodium hydroxide $(\mathrm{NaOH})$, and sodium hexametaphosphate $\left(\mathrm{Na}_{6} \mathrm{P}_{6} \mathrm{O}_{18}\right)$.

With the temperature factor (since extinguishing the fires involves a high ambient temperature) and the presence of several components in the mixture, for example $(36,37,39)$, some products may also be released into the environment:

$3 \mathrm{Al}_{2}(\mathrm{OH})_{5} \mathrm{Cl} \rightarrow \mathrm{AlCl}_{3}+5 \mathrm{Al}(\mathrm{OH})_{3}$

in turn, when exposed to heat:

$2 \mathrm{Al}(\mathrm{OH})_{3} \rightarrow \mathrm{Al}_{2} \mathrm{O}_{3}+3 \mathrm{H}_{2} \mathrm{O}_{(\mathrm{g})}(2)$

Also, the temperature factor can lead to the formation of such products for the mixture:

$3 \mathrm{Al}_{2}(\mathrm{OH})_{5} \mathrm{Cl}+6\left(\mathrm{NH}_{4}\right)_{2} \mathrm{SO}_{4} \rightarrow 2 \mathrm{Al}_{2}\left(\mathrm{SO}_{4}\right)_{3}+\mathrm{Al}_{2} \mathrm{O}_{3}$

$+12 \mathrm{NH}_{3}(\mathrm{~g})+3 \mathrm{HCl}(\mathrm{g})+12 \mathrm{H}_{2} \mathrm{O}_{(\mathrm{g})}(3)$

In the composition of foams, these compounds can be found mainly in aquatic and soil ecosystems. The paper examined the effect of inorganic components of foaming agents on aquatic ecosystems. Since sodium hexametaphosphate is a more branched structure of sodium metaphosphate, the main analysis can be done by using EXA data on sodium metaphosphate.

The data on the predicted safe concentration (PNEC) of a substance for organisms living in marine and freshwater ecosystems are presented in Table 1.

It was concluded that the lower the PNEC value, the unsafer the substance for organisms, so aluminum and ammonium sulfates are more dangerous to get into fresh water, and aluminum sulfate and sulfamic acid to get into the sea water, 
periodic discharges, containing in the fresh water, sulfamic acid and ammonium sulfate are unsafer. In sediments, the accumulation of ammonium sulfate (fresh water) and sulfamic acid (seawater) is unsafe. That is, as seen from the presented PNEC values, sulfonated inorganic compounds have a greater negative effect on aquatic organisms.' Thus, the comparative analysis of the environmental hazard of the tested inorganic additives of foaming agents showed that the most dangerous for the environment are aluminum compounds and sulfamic acid, and the safest are magnesium and sodium chlorides.

To assess the environmental hazard to aquatic organisms data, obtained in the same conditions, were used.

For a comparative analysis of inorganic additives of foaming agents according to their short-term toxicity the parameter LC50 (4 days) is the most suitable for fish. Table 2 shows sodium, calcium and magnesium chlorides, as well as sodium bicarbonate in the short term are the least toxic for fish. It is difficult to name the most toxic substance for fish, since there is data variability. If we accept that in the case of variable data, we follow the lowest value, then the most dangerous compounds are aluminum-based compounds for fish. If we take the maximum values of LC50 (4 days), then sulfamic acid, ammonium sulfate, and sodium hexametaphosphate are more toxic in the short term for fish.

Values of long-term toxicity of substances for fish also vary greatly, and the data themselves are incomplete. So, NOEC for the studied compounds is presented to the fullest extent possible (Table 2 ). It can be noted that sodium chloride is the safest for freshwater fish in terms of long-term toxicity, and the most dangerous is basic aluminum chloride and, as a possible reaction product, aluminum sulfate. 
Table 1. PNEC for Aquatic Organisms, mg/L (mg/kg sediment $\mathrm{dw}$ )

\begin{tabular}{|c|c|c|c|c|c|c|}
\hline \multirow[t]{2}{*}{ Substance } & \multicolumn{6}{|c|}{ PNEC } \\
\hline & Freshwater & $\begin{array}{l}\text { Intermittent releases } \\
\text { (freshwater) }\end{array}$ & Marine water & $\begin{array}{l}\text { Sewage } \\
\text { treatment plant }\end{array}$ & $\begin{array}{l}\text { Sediment } \\
\text { (freshwater) }\end{array}$ & $\begin{array}{l}\text { Sediment } \\
\text { (marine water) }\end{array}$ \\
\hline Magnesium chloride & $3.21 \mathrm{mg} / \mathrm{L}$ & $5.48 \mathrm{mg} / \mathrm{L}$ & $320 \mu \mathrm{g} / \mathrm{L}$ & $90 \mathrm{mg} / \mathrm{L}$ & $\begin{array}{l}288.9 \mathrm{mg} / \mathrm{kg} \text { sediment } \\
\mathrm{dw}\end{array}$ & $\begin{array}{l}28.89 \mathrm{mg} / \mathrm{kg} \\
\text { sediment dw }\end{array}$ \\
\hline Sodium chloride & $5 \mathrm{mg} / \mathrm{L}$ & No data & $\begin{array}{l}\text { aquatic toxicity } \\
\text { unlikely }\end{array}$ & $\begin{array}{l}\text { aquatic toxicity } \\
\text { unlikely }\end{array}$ & $\begin{array}{l}\text { No exposure of } \\
\text { sediment expected }\end{array}$ & $\begin{array}{l}\text { No exposure of } \\
\text { sediment expected }\end{array}$ \\
\hline Calcium chloride & No data & No data & No data & No data & No data & No data \\
\hline $\begin{array}{l}\text { Basic Aluminum } \\
\text { chloride }\end{array}$ & $\begin{array}{l}\text { No hazard } \\
\text { identified }\end{array}$ & No hazard identified & $\begin{array}{l}\text { No hazard } \\
\text { identified }\end{array}$ & $\begin{array}{l}\text { No hazard } \\
\text { identified }\end{array}$ & No hazard identified & $\begin{array}{l}\text { No hazard } \\
\text { identified }\end{array}$ \\
\hline Sodium hydroxide & No data & No data & No data & No data & No data & No data \\
\hline Sulfamic acid & $1.8 \mathrm{mg} / \mathrm{L}$ & $480 \mu \mathrm{g} / \mathrm{L}$ & $180 \mu \mathrm{g} / \mathrm{L}$ & No data & $\begin{array}{l}8.36 \mathrm{mg} / \mathrm{kg} \text { sediment } \\
\mathrm{dw}\end{array}$ & $\begin{array}{l}840 \mathrm{mg} / \mathrm{kg} \\
\text { sediment dw }\end{array}$ \\
\hline Ammonium sulfate & $312 \mu \mathrm{g} / \mathrm{L}$ & $530 \mu \mathrm{g} / \mathrm{L}$ & $31.2 \mu \mathrm{g} / \mathrm{L}$ & $16.18 \mathrm{mg} / \mathrm{L}$ & $63 \mathrm{mg} / \mathrm{kg}$ sediment $\mathrm{dw}$ & No data \\
\hline Sodium bicarbonate & No data & No data & No data & No data & No data & No data \\
\hline Sodium carbonate & $\begin{array}{l}\text { aquatic toxicity } \\
\text { unlikely }\end{array}$ & aquatic toxicity unlikely & $\begin{array}{l}\text { aquatic toxicity } \\
\text { unlikely }\end{array}$ & $\begin{array}{l}\text { aquatic toxicity } \\
\text { unlikely }\end{array}$ & No data & No data \\
\hline $\begin{array}{l}\text { Sodium } \\
\text { hexametaphosphate }\end{array}$ & $\begin{array}{l}\text { No hazard } \\
\text { identified }\end{array}$ & No hazard identified & $\begin{array}{l}\text { No hazard } \\
\text { identified }\end{array}$ & $\begin{array}{l}\text { No hazard } \\
\text { identified }\end{array}$ & No hazard identified & $\begin{array}{l}\text { No hazard } \\
\text { identified }\end{array}$ \\
\hline Aluminum chloride & $\begin{array}{l}\text { No hazard } \\
\text { identified }\end{array}$ & No hazard identified & $\begin{array}{l}\text { No hazard } \\
\text { identified }\end{array}$ & No data & No data & No data \\
\hline Aluminum sulfate & $\begin{array}{l}300-4500 \\
000 \mathrm{ng} / \mathrm{L}\end{array}$ & $30.11 \mathrm{mg} / \mathrm{L}$ & $\begin{array}{l}30-64000000 \\
\mathrm{ng} / \mathrm{L}\end{array}$ & $\begin{array}{l}\text { No hazard } \\
\text { identified }\end{array}$ & $10 \mathrm{mg} / \mathrm{kg}$ sediment $\mathrm{dw}$ & $\begin{array}{l}31.4 \mathrm{mg} / \mathrm{kg} \\
\text { sediment dw }\end{array}$ \\
\hline Aluminum oxide & $\begin{array}{l}\text { aquatic toxicity } \\
\text { unlikely }\end{array}$ & aquatic toxicity unlikely & $\begin{array}{l}\text { aquatic toxicity } \\
\text { unlikely }\end{array}$ & $\begin{array}{l}\text { aquatic toxicity } \\
\text { unlikely }\end{array}$ & No data & No data \\
\hline Aluminum hydroxide & $\begin{array}{l}\text { No hazard } \\
\text { identified }\end{array}$ & No hazard identified & $\begin{array}{l}\text { No hazard } \\
\text { identified }\end{array}$ & $\begin{array}{l}\text { No hazard } \\
\text { identified }\end{array}$ & No data & No data \\
\hline
\end{tabular}

Table 2. Data on toxicity of test substances for aquatic organisms

$\begin{array}{lll}\text { Substance } & \begin{array}{l}\text { Short-term toxicity to } \\ \text { fish }\end{array} & \begin{array}{l}\text { Long-term } \\ \text { toxicity to fish }\end{array}\end{array}$

1

1

chloride 2 3 LC50 (4 days) 541 - $\quad$ No data $2119.3 \mathrm{mg} / \mathrm{L}$ Summaries:

Short-term toxicity to aquatic invertebrates 4

LC50 (48 h) 140 $548.4 \mathrm{mg} / \mathrm{L}$ Summaries:
Long-term toxicity

to aquatic invertebrates 5

EC10 (21 days) 82 $321 \mathrm{mg} / \mathrm{L}$

Summaries:
Toxicity to aquatic algae and cyanobacteria

EC50 (72 h) 100 $\mathrm{mg} / \mathrm{L}$ NOEC (72 h) 100 $\mathrm{mg} / \mathrm{L}$
Toxicity to microorganisms 7 EC50 (3 h) 900 $\mathrm{mg} / \mathrm{L}$ Summaries: 


\begin{tabular}{|c|c|c|c|c|c|c|}
\hline & $\begin{array}{l}\text { LC50 for freshwater fish } \\
2,119 \mathrm{~g} / \mathrm{L} \\
\text { LC50 for marine water } \\
\text { fish } 10,968 \mathrm{~g} / \mathrm{L}\end{array}$ & & $\begin{array}{l}\text { EC50/LC50 for } \\
\text { freshwater } \\
\text { invertebrates } 548,4 \\
\mathrm{mg} / \mathrm{L} \\
\text { EC50/LC50 for marine } \\
\text { invertebrates } 3,259 \mathrm{~g} / \\
\mathrm{L}\end{array}$ & $\begin{array}{l}\text { EC10/LC10 or NOEC } \\
\text { for freshwater } \\
\text { invertebrates } \\
321 \mathrm{mg} / \mathrm{L}\end{array}$ & $\begin{array}{l}\text { Summaries: } \\
\text { EC10 or NOEC for } \\
\text { freshwater algae } \\
100 \mathrm{mg} / \mathrm{L}\end{array}$ & $\begin{array}{l}\text { EC10 or NOEC for } \\
\text { microorganisms } \\
900 \mathrm{mg} / \mathrm{L}\end{array}$ \\
\hline $\begin{array}{l}\text { Sodium } \\
\text { chloride }\end{array}$ & $\begin{array}{l}\text { LC50 (4 days) } 5.84 \mathrm{~g} / \mathrm{L} \\
\text { Summaries: } \\
\text { LC50 for freshwater fish } \\
5.84 \mathrm{~g} / \mathrm{L}\end{array}$ & $\begin{array}{l}\text { NOEC ( } 33 \text { days) } 252 \\
-533 \mathrm{mg} / \mathrm{L} \\
\text { LOEC ( } 33 \text { days) } \\
352-734 \mathrm{mg} / \mathrm{L} \\
\text { Summaries: } \\
\text { EC10/LC10 or NOEC } \\
\text { for freshwater fish } \\
252 \mathrm{mg} / \mathrm{L}\end{array}$ & $\begin{array}{l}\text { Summaries: } \\
\text { EC50/LC50 for } \\
\text { freshwater } \\
\text { invertebrates } \\
1.9 \mathrm{~g} / \mathrm{L}\end{array}$ & $\begin{array}{l}\text { NOEC ( } 21 \text { days) } 314 \\
\mathrm{mg} / \mathrm{L} \\
\text { LOEC ( } 21 \text { days) } 441 \\
\mathrm{mg} / \mathrm{L} \\
\text { Summaries: } \\
\text { EC10/LC10 or NOEC } \\
\text { for freshwater } \\
\text { invertebrates } \\
314 \mathrm{mg} / \mathrm{L}\end{array}$ & $\begin{array}{l}\text { EC50 (5 days) } 2.43 \\
\mathrm{~g} / \mathrm{L} \\
\text { Summaries: } \\
\text { EC50 for freshwater } \\
\text { algae } \\
2.43 \mathrm{~g} / \mathrm{L}\end{array}$ & $\begin{array}{l}\text { EC50 (4 days) } \\
6.87 \mathrm{~g} / \mathrm{L} \\
\text { Summaries: } \\
\text { EC10 or NOEC for } \\
\text { microorganisms } \\
5 \mathrm{~g} / \mathrm{L}\end{array}$ \\
\hline 1 & 2 & 3 & 4 & $\mathbf{5}$ & 6 & $\mathbf{7}$ \\
\hline $\begin{array}{l}\text { Calcium } \\
\text { chloride }\end{array}$ & $\begin{array}{l}\text { LC50 (4 days) } 4.63 \mathrm{~g} / \mathrm{L} \\
\text { LC50 }(48 \mathrm{~h}) 6.56 \mathrm{~g} / \mathrm{L} \\
\text { LC50 }(24 \mathrm{~h}) 6.66 \mathrm{~g} / \mathrm{L}\end{array}$ & No data & $\begin{array}{l}\text { LC50 }(48 \mathrm{~h}) \\
2.4-2.77 \mathrm{~g} / \mathrm{L} \\
\operatorname{NOEC}(48 \mathrm{~h}) \\
2 \mathrm{~g} / \mathrm{L}\end{array}$ & $\begin{array}{l}\text { EC50 (21 days) } 610 \\
\mathrm{mg} / \mathrm{L} \\
\text { LC50 (21 days) } 330- \\
920 \mathrm{mg} / \mathrm{L}\end{array}$ & $\begin{array}{l}\text { EC50 (72 h) } 2.9- \\
27 \mathrm{~g} / \mathrm{L} \\
\text { EC20 }(72 \mathrm{~h}) 1 \mathrm{~g} / \mathrm{L}\end{array}$ & No data \\
\hline $\begin{array}{l}\text { Basic aluminum } \\
\text { chloride }\end{array}$ & $\begin{array}{l}\text { LC50 (4 days) } 1.39-186 \\
\mathrm{mg} / \mathrm{L} \\
\mathrm{LC} 10 \text { (4 days) } 580-142 \\
000 \mu \mathrm{g} / \mathrm{L} \\
\text { EC50 ( } 4 \text { days) } 156 \mu \mathrm{g} / \mathrm{L} \\
\text { NOEC (4 days) } 156-1 \\
000000 \mu \mathrm{g} / \mathrm{L}\end{array}$ & $\begin{array}{l}\text { NOEC (60 days) } 13-26 \\
\mu \mathrm{g} / \mathrm{L} \\
\text { NOEC ( } 7 \text { days) } 752-56 \\
480 \mu \mathrm{g} / \mathrm{L} \\
\text { LOEC ( } 7 \text { days) } 831-91 \\
420 \mu \mathrm{g} / \mathrm{L} \\
\text { LC50 (42 days) } 15 \mu \mathrm{g} / \mathrm{L} \\
\text { LC50 ( } 28 \text { days) } 19 \mu \mathrm{g} / \mathrm{L}\end{array}$ & $\begin{array}{l}\text { EC50 (48 h) } \\
214-200000 \\
\mu g / L \\
\text { EC10 }(48 \mathrm{~h}) 2.8 \\
-42 \mathrm{mg} / \mathrm{L} \\
\operatorname{NOEC~}(48 \mathrm{~h}) \\
160 \mathrm{mg} / \mathrm{L}\end{array}$ & $\begin{array}{l}\text { NOEC (7 days) } 15 \\
\mathrm{mg} / \mathrm{L} \\
\mathrm{LOEC}(7 \text { days }) 15 \mathrm{mg} / \\
\mathrm{L}\end{array}$ & $\begin{array}{l}\text { EC50 }(72 \mathrm{~h}) 75-14 \\
000 \mu \mathrm{g} / \mathrm{L} \\
\mathrm{NOEC}(72 \mathrm{~h}) 20-1 \\
000 \mu \mathrm{g} / \mathrm{L} \\
\text { EC10 (72 h) } 15-3 \\
100 \mu \mathrm{g} / \mathrm{L}\end{array}$ & $\begin{array}{l}\text { EC50 }(3 \mathrm{~h}) 4.4-1 \\
000 \mathrm{mg} / \mathrm{L} \\
\text { EC10 }(3 \mathrm{~h}) 4.4- \\
1000 \mathrm{mg} / \mathrm{L}\end{array}$ \\
\hline $\begin{array}{l}\text { Sodium } \\
\text { hydroxide }\end{array}$ & No data & No data & $\begin{array}{l}\text { EC50 }(48 \mathrm{~h}) \\
40.4 \mathrm{mg} / \mathrm{L}\end{array}$ & No data & No data & No data \\
\hline Sulfamic acid & $\begin{array}{l}\text { LC50 (4 days) } 70.3 \mathrm{mg} / \mathrm{L} \\
\text { Summaries: } \\
\text { LC50 for freshwater fish }\end{array}$ & $\begin{array}{l}\text { NOEC (65 days) } 25 \mu \mathrm{g} / \\
\mathrm{L} \\
\text { NOEC (34 days) } 60 \mathrm{mg} / \\
\mathrm{L}\end{array}$ & $\begin{array}{l}\text { EC50 }(48 \mathrm{~h}) \\
71.6 \mathrm{mg} / \mathrm{L} \\
\text { EC50 }(24 \mathrm{~h}) \\
71.6 \mathrm{mg} / \mathrm{L}\end{array}$ & $\begin{array}{l}\text { NOEC (35 days) } 150 \\
\mu \mathrm{g} / \mathrm{L} \\
\text { NOEC (21 days) } 19 \\
\mathrm{mg} / \mathrm{L}\end{array}$ & $\begin{array}{l}\text { EC50 (72 h) } 33.8- \\
48 \mathrm{mg} / \mathrm{L} \\
\text { NOEC }(72 \mathrm{~h}) 18 \\
\mathrm{mg} / \mathrm{L}\end{array}$ & $\begin{array}{l}\text { EC50 }(3 \text { h) } 200 \\
\mathrm{mg} / \mathrm{L} \\
\mathrm{NOEC}(3 \mathrm{~h}) 200 \\
\mathrm{mg} / \mathrm{L}\end{array}$ \\
\hline
\end{tabular}




\begin{tabular}{|c|c|c|c|c|c|c|}
\hline & $70.3 \mathrm{mg} / \mathrm{L}$ & $\begin{array}{l}\text { Summaries: } \\
\text { EC } 10 / \mathrm{LC} 10 \text { or NOEC for } \\
\text { freshwater fish } 60 \mathrm{mg} / \mathrm{L}\end{array}$ & $\begin{array}{l}\text { Summaries: } \\
\text { EC50/LC50 for } \\
\text { freshwater } \\
\text { invertebrates } \\
71.6 \mathrm{mg} / \mathrm{L}\end{array}$ & $\begin{array}{l}\text { LOEC ( } 21 \text { days) } 34 \\
\text { mg/L } \\
\text { EC50 ( } 21 \text { days) } 60 \\
\text { mg/L } \\
\text { Summaries: } \\
\text { EC10/LC10 or NOEC } \\
\text { for freshwater } \\
\text { invertebrates } \\
19 \text { mg/L }\end{array}$ & $\begin{array}{l}\text { EC10 }(72 \mathrm{~h}) 13.3- \\
29.5 \mathrm{mg} / \mathrm{L} \\
\text { Summaries: } \\
\text { EC50 or freshwater } \\
\text { algae } \\
48 \mathrm{mg} / \mathrm{L} \\
\text { EC10 or NOEC for } \\
\text { freshwater algae } \\
18 \mathrm{mg} / \mathrm{L}\end{array}$ & $\begin{array}{l}\text { Summaries: } \\
\text { EC50 for } \\
\text { microorganisms } \\
200 \mathrm{mg} / \mathrm{L} \\
\text { EC10 or NOEC for } \\
\text { microorganisms } \\
200 \mathrm{mg} / \mathrm{L}\end{array}$ \\
\hline 1 & 2 & 3 & 4 & 5 & 6 & 7 \\
\hline $\begin{array}{l}\text { Ammonium } \\
\text { sulfate }\end{array}$ & $\begin{array}{l}\text { LC50 (4 days) } 53-57.2 \\
\mathrm{mg} / \mathrm{L} \\
\text { Summaries: } \\
\text { LC50 for freshwater fish } \\
53 \mathrm{mg} / \mathrm{L}\end{array}$ & $\begin{array}{l}\text { EC10 (30 days) } 5.29 \\
\mathrm{mg} / \mathrm{L} \\
\text { Summaries: } \\
\text { EC10/LC10 or NOEC for } \\
\text { freshwater fish } 5.29 \\
\mathrm{mg} / \mathrm{L}\end{array}$ & $\begin{array}{l}\text { EC50 (48 h) } \\
121.7-169 \\
\mathrm{mg} / \mathrm{L} \\
\text { Summaries: } \\
\text { EC50/LC50 for } \\
\text { freshwater } \\
\text { invertebrates } \\
169 \mathrm{mg} / \mathrm{L}\end{array}$ & $\begin{array}{l}\text { EC10 (70 days) } 3.12 \\
\mathrm{mg} / \mathrm{L} \\
\text { Summaries: } \\
\text { EC10/LC10 or NOEC } \\
\text { for freshwater } \\
\text { invertebrates } \\
3.12 \mathrm{mg} / \mathrm{L}\end{array}$ & $\begin{array}{l}\text { EC50 (18 days) } 2.7 \\
\text { g/L } \\
\text { EC50 (5 days) } \\
1.605 \mathrm{~g} / \mathrm{L}\end{array}$ & $\begin{array}{l}\text { EC50 (30 min) } \\
1.618 \mathrm{~g} / \mathrm{L}\end{array}$ \\
\hline $\begin{array}{l}\text { Sodium } \\
\text { bicarbonate }\end{array}$ & $\begin{array}{l}\text { LC50 (4 days) } 7.1 \mathrm{~g} / \mathrm{L} \\
\text { NOEC (4 days) } 5.2 \mathrm{~g} / \mathrm{L} \\
\text { Summaries: } \\
\text { LC50 for freshwater fish } \\
7.1 \mathrm{~g} / \mathrm{L}\end{array}$ & No data & $\begin{array}{l}\text { EC50 (48 h) } 4.1 \\
\mathrm{~g} / \mathrm{L} \\
\text { NOEC }(48 \mathrm{~h}) \\
3.1 \mathrm{~g} / \mathrm{L} \\
\text { Summaries: } \\
\text { EC50 / LC50 for } \\
\text { freshwater } \\
\text { invertebrates } \\
4.1 \mathrm{~g} / \mathrm{L}\end{array}$ & $\begin{array}{l}\text { NOEC (21 days) } 576 \\
\mathrm{mg} / \mathrm{L}\end{array}$ & No data & No data \\
\hline $\begin{array}{l}\text { Sodium } \\
\text { carbonate }\end{array}$ & $\begin{array}{l}\text { LC50 (4 days) } 300 \mathrm{mg} / \mathrm{L} \\
\text { Summaries: } \\
\text { LC50 for freshwater fish } \\
300 \mathrm{mg} / \mathrm{L}\end{array}$ & No data & $\begin{array}{l}\text { EC50 }(48 \mathrm{~h}) \\
200-227 \mathrm{mg} / \mathrm{L} \\
\text { Summaries: } \\
\text { EC50/LC50 for } \\
\text { freshwater } \\
\text { invertebrates } \\
200 \mathrm{mg} / \mathrm{L}\end{array}$ & No data & No data & No data \\
\hline
\end{tabular}




\begin{tabular}{|c|c|c|c|c|c|c|}
\hline 1 & 2 & 3 & 4 & 5 & 6 & 7 \\
\hline $\begin{array}{l}\text { Sodium } \\
\text { hexametaphosp } \\
\text { hate }\end{array}$ & $\begin{array}{l}\text { LC50 (4 days) } 100 \\
\text { mg/L } \\
\text { NOEC (4 days) } 100 \\
\text { mg/L } \\
\text { Summaries: } \\
\text { LC50 for freshwater } \\
\text { fish } 100 \mathrm{mg} / \mathrm{L}\end{array}$ & Vo data & $\begin{array}{l}\text { EC50 }(48 \mathrm{~h}) 485 \\
\mathrm{mg} / \mathrm{L} \\
\text { Summaries: } \\
\text { EC50/LC50 for } \\
\text { freshwater } \\
\text { invertebrates } \\
100 \mathrm{mg} / \mathrm{L}\end{array}$ & No data & $\begin{array}{l}\text { EC50 }(72 \mathrm{~h}) 100 \\
\mathrm{mg} / \mathrm{L} \\
\mathrm{NOEC}(72 \mathrm{~h}) 32 \\
\mathrm{mg} / \mathrm{L} \text { LOEC }(72 \mathrm{~h}) \\
100 \mathrm{mg} / \mathrm{L} \\
\text { Summaries: } \\
\text { EC50 for freshwater } \\
\text { algae } \\
100 \mathrm{mg} / \mathrm{L}\end{array}$ & $\begin{array}{l}\text { EC50 }(3 \mathrm{~h}) 1 \mathrm{~g} / \mathrm{L} \\
\text { NOEC }(3 \mathrm{~h}) 1 \mathrm{~g} / \mathrm{L} \\
\text { Summaries: } \\
\text { EC50 for } \\
\text { microorganisms } \\
1 \mathrm{~g} / \mathrm{L} \\
\text { EC10 or NOEC for } \\
\text { microorganisms } \\
1 \mathrm{~g} / \mathrm{L}\end{array}$ \\
\hline $\begin{array}{l}\text { Aluminum } \\
\text { chloride }\end{array}$ & $\begin{array}{l}\text { LC50 (16 days) } 430 \\
-3910 \mu \mathrm{g} / \mathrm{L} \\
\text { LC50 ( } 8 \text { days) } 22.4 \\
\mathrm{mg} / \mathrm{L} \\
\text { LC50 (4 days) } 78 \text { - } \\
218640 \mu \mathrm{g} / \mathrm{L} \\
\text { LC50 }(72 \mathrm{~h}) 10- \\
19.3 \mathrm{mg} / \mathrm{L} \\
\text { LC50 (48 h) } 11.5 \\
\mathrm{mg} / \mathrm{L}\end{array}$ & $\begin{array}{l}\operatorname{VOEC}(60 \text { days) } 88-350 \\
\text { ug/L } \\
\text { NOEC (30 days) } 57-88 \\
\text { Lg/L } \\
\text { NOEC ( } 28 \text { days) } 4.7- \\
23.1 \mathrm{mg} / \mathrm{L} \\
\text { NOEC ( } 7 \text { days) } 160-56 \\
480 \mu \mathrm{g} / \mathrm{L} \\
\text { _OEC (60 days) } 169- \\
350 \mu \mathrm{g} / \mathrm{L}\end{array}$ & $\begin{array}{l}\text { EC50 (48 h) } 1.5 \text { - } \\
27.3 \mathrm{mg} / \mathrm{L} \\
\text { LC50 (4 days) } 22 \text { - } \\
30.6 \mathrm{mg} / \mathrm{L} \\
\text { LC50 }(48 \mathrm{~h}) 71- \\
99600 \mu \mathrm{g} / \mathrm{L} \\
\text { NOEC }(4 \mathrm{days}) \\
22.6 \mathrm{mg} / \mathrm{L} \\
\text { NOEC }(48 \mathrm{~h}) 5- \\
672 \mu \mathrm{g} / \mathrm{L}\end{array}$ & $\begin{array}{l}\text { NOEC ( } 28 \text { days) } 1.89 \\
\mathrm{mg} / \mathrm{L} \\
\mathrm{NOEC}(21 \text { days) } 76 \text { - } \\
137 \mu \mathrm{g} / \mathrm{L} \\
\mathrm{NOEC} \mathrm{(} 8 \text { days) } 4.9 \\
\mathrm{mg} / \mathrm{L} \\
\mathrm{NOEC}(7 \text { days) } 1.1- \\
1.4 \mathrm{mg} / \mathrm{L} \\
\mathrm{NOEC}(6 \text { days) } 340- \\
1020 \mu \mathrm{g} / \mathrm{L}\end{array}$ & $\begin{array}{l}\text { EC50 (4 days) } 24- \\
570 \mu \mathrm{g} / \mathrm{L} \\
\text { EC50 (72 h) } 200-4 \\
980 \mu \mathrm{g} / \mathrm{L} \\
\text { NOEC }(72 \mathrm{~h}) 4- \\
600 \mu \mathrm{g} / \mathrm{L} \\
\text { LOEC }(72 \mathrm{~h}) 1 \mathrm{mg} / \mathrm{L} \\
\text { EC10 (72 h) } 51-3 \\
155 \mu \mathrm{g} / \mathrm{L}\end{array}$ & No data \\
\hline 1 & 2 & 3 & 4 & 5 & 6 & 7 \\
\hline $\begin{array}{l}\text { Aluminum } \\
\text { sulfate }\end{array}$ & $\begin{array}{l}\text { LC50 (8 days) } 122.17 \text { - } \\
161.4 \mathrm{mg} / \mathrm{L} \\
\text { LC50 ( } 7 \text { days) } 430- \\
4270 \mu \mathrm{g} / \mathrm{L} \\
\text { LC50 (6 days) } 560- \\
6650 \mu \mathrm{g} / \mathrm{L} \\
\text { LC50 ( } 5.833 \text { days) } 22.74 \\
\mathrm{mg} / \mathrm{L} \\
\text { LC50 ( } 5 \text { days) } 1.05- \\
20.8 \text { mg/L } \\
\text { Summaries: } \\
\text { LC50 for freshwater fish }\end{array}$ & $\begin{array}{l}\text { NOEC (60 days) } 13-1 \\
670 \mu \mathrm{g} / \mathrm{L} \\
\text { NOEC ( } 33 \text { days) } 71.5- \\
558.1 \mu \mathrm{g} / \mathrm{L} \\
\text { NOEC ( } 30 \text { days) } 250-1 \\
670 \mu \mathrm{g} / \mathrm{L} \\
\text { NOEC ( } 28 \text { days) } 29.8- \\
44.9 \mathrm{mg} / \mathrm{L} \\
\text { NOEC ( } 15 \text { days) } 1.67 \\
\text { mg/L } \\
\text { Summaries: } \\
\text { EC10/LC10 or NOEC for } \\
\text { freshwater fish } 44.9 \\
\text { mg/L }\end{array}$ & $\begin{array}{l}\text { EC50 (4 days) } \\
5.9-58.2 \mathrm{mg} / \mathrm{L} \\
\text { EC50 (72 h) } \\
27.7 \mathrm{mg} / \mathrm{L} \\
\text { EC50 }(48 \mathrm{~h}) 1.4 \\
-200 \mathrm{mg} / \mathrm{L} \\
\text { LC50 (7 days) } \\
11.2 \mathrm{mg} / \mathrm{L} \\
\text { LC50 (72 h) } \\
1.52-19.5 \mathrm{mg} / \\
\mathrm{L} \\
\text { Summaries: } \\
\text { EC50/LC50 for } \\
\text { freshwater }\end{array}$ & $\begin{array}{l}\text { NOEC ( } 42 \text { days) } 232.6 \\
-453.8 \mu \mathrm{g} / \mathrm{L} \\
\text { NOEC ( } 30 \text { days) } 1.092 \\
-2.099 \mathrm{mg} / \mathrm{L} \\
\text { NOEC ( } 28 \text { days) } 53.1 \\
-12000 \mu \mathrm{g} / \mathrm{L} \\
\text { NOEC ( } 17 \text { days) } 962.5 \\
\mu \mathrm{g} / \mathrm{L} \\
\text { NOEC (10 days) } 1.1 \text { - } \\
4.282 \mathrm{mg} / \mathrm{L} \\
\text { Summaries: } \\
\text { EC10/LC10 or NOEC } \\
\text { for freshwater } \\
\text { invertebrates }\end{array}$ & $\begin{array}{l}\text { EC50 (30 days) } \\
1.767 \mathrm{~g} / \mathrm{L} \\
\text { EC50 (5 days) } 3.011 \\
-19.091 \mathrm{~g} / \mathrm{L} \\
\text { EC50 (4 days) } 460- \\
570 \mu \mathrm{g} / \mathrm{L} \\
\text { EC50 }(72 \mathrm{~h}) 40- \\
100000 \mu \mathrm{g} / \mathrm{L} \\
\text { EC50 }(22 \mathrm{~h}) 25 \mathrm{mg} / \\
\mathrm{L}\end{array}$ & $\begin{array}{l}\text { EC50 (1.084 } \\
\text { years) } 500-3 \\
100 \mu \mathrm{g} / \mathrm{L} \\
\text { EC50 (22 days) } \\
114-512 \mu \mathrm{g} / \mathrm{L} \\
\text { EC50 (5 days) } \\
3.011-19.091 \mathrm{~g} / \\
\mathrm{L} \\
\text { EC50 }(24 \mathrm{~h}) 6 \\
\mathrm{mg} / \mathrm{L} \\
\text { EC50 }(3 \mathrm{~h}) 200- \\
1000 \mathrm{mg} / \mathrm{L} \\
\text { Summaries: }\end{array}$ \\
\hline
\end{tabular}




\begin{tabular}{|c|c|c|c|c|c|c|}
\hline & $\begin{array}{l}122.17 \mathrm{mg} / \mathrm{L} \\
\text { LC50 for marine water } \\
\text { fish } 12.2 \mathrm{mg} / \mathrm{L}\end{array}$ & $\begin{array}{l}\text { EC10/LC10 or NOEC for } \\
\text { marine water fish } 4.5 \\
\mathrm{mg} / \mathrm{L}\end{array}$ & $\begin{array}{l}\text { invertebrates } \\
242 \mathrm{mg} / \mathrm{L} \\
\text { EC50/LC50 for } \\
\text { marine } \\
\text { invertebrates } \\
19.5 \mathrm{mg} / \mathrm{L}\end{array}$ & $\begin{array}{l}12 \mathrm{mg} / \mathrm{L} \\
\text { EC10/LC10 or NOEC } \\
\text { for marine } \\
\text { invertebrates } \\
41.2 \mathrm{mg} / \mathrm{L}\end{array}$ & $\begin{array}{l}\text { algae } \\
3.011 \mathrm{~g} / \mathrm{L} \\
\text { EC50 for marine } \\
\text { algae } \\
302 \mathrm{mg} / \mathrm{L} \\
\text { EC10 or NOEC for } \\
\text { freshwater algae } \\
602 \mathrm{mg} / \mathrm{L} \\
\text { EC10 or NOEC for } \\
\text { marine algae } \\
30 \mathrm{mg} / \mathrm{L}\end{array}$ & $\begin{array}{l}\text { EC50 for } \\
\text { microorganisms } \\
3.011 \mathrm{~g} / \mathrm{L} \\
\text { EC10 or NOEC for } \\
\text { microorganisms } \\
200-602 \mathrm{mg} / \mathrm{L}\end{array}$ \\
\hline 1 & $\mathbf{2}$ & $\mathbf{3}$ & 4 & 5 & 6 & 7 \\
\hline $\begin{array}{l}\text { Aluminum } \\
\text { oxide }\end{array}$ & $\begin{array}{l}\text { LC50 (16 days) } 430-3 \\
910 \mu \mathrm{g} / \mathrm{L} \text { LC50 (8 days) } \\
22.4 \mathrm{mg} / \mathrm{L} \\
\text { LC50 ( } 4 \text { days) } 78-218 \\
644.1 \mu \mathrm{g} / \mathrm{L} \\
\text { LC50 (4 days) } 2.9 \mu \mathrm{mol} / \mathrm{L} \\
\text { LC50 (72 h) } 10-19.3 \\
\mathrm{mg} / \mathrm{L}\end{array}$ & $\begin{array}{l}\text { NOEC (60 days) } 88- \\
350 \mu \mathrm{g} / \mathrm{L} \\
\text { NOEC ( } 33 \text { days) } 71.5 \text { - } \\
558.1 \mu \mathrm{g} / \mathrm{L} \\
\text { NOEC ( } 30 \text { days) } 57-88 \\
\mu \mathrm{g} / \mathrm{L} \\
\text { NOEC ( } 28 \text { days) } 4.7- \\
23.1 \mathrm{mg} / \mathrm{L} \\
\text { NOEC ( } 7 \text { days) } 25.1- \\
56480 \mu \mathrm{g} / \mathrm{L}\end{array}$ & $\begin{array}{l}\text { EC50 }(48 \mathrm{~h}) 1.5 \\
-2.56 \mathrm{mg} / \mathrm{L} \\
\text { LC50 (4 days) } \\
22-30.6 \mathrm{mg} / \mathrm{L} \\
\text { LC50 }(48 \mathrm{~h}) 5.7 \\
-99600 \mu \mathrm{g} / \mathrm{L} \\
\mathrm{NOEC}(4 \mathrm{days}) \\
22.6 \mathrm{mg} / \mathrm{L} \\
\mathrm{NOEC}(48 \mathrm{~h}) 5- \\
672 \mu \mathrm{g} / \mathrm{L}\end{array}$ & $\begin{array}{l}\text { NOEC (42 days) } 232.6 \\
-453.8 \mu \mathrm{g} / \mathrm{L} \\
\text { NOEC ( } 30 \text { days) } 1.092 \\
-2.099 \mathrm{mg} / \mathrm{L} \\
\text { NOEC ( } 28 \text { days) } 53.1 \\
-4281.8 \mu \mathrm{g} / \mathrm{L} \\
\text { NOEC }(21 \text { days) } 76- \\
600 \mu \mathrm{g} / \mathrm{L} \\
\text { NOEC (17 days) } 962.5 \\
\mu \mathrm{g} / \mathrm{L}\end{array}$ & $\begin{array}{l}\text { EC50 (4 days) } 5.4- \\
570 \mu \mathrm{g} / \mathrm{L} \\
\text { EC50 (72 h) } 16.9-4 \\
980 \mu \mathrm{g} / \mathrm{L} \\
\text { NOEC }(72 \mathrm{~h}) 4-600 \\
\mu \mathrm{g} / \mathrm{L} \\
\text { LOEC }(72 \mathrm{~h}) 400-1 \\
000 \mu \mathrm{g} / \mathrm{L} \\
\text { EC10 (72 h) } 203-3 \\
155000 \mathrm{ng} / \mathrm{L}\end{array}$ & No data \\
\hline $\begin{array}{l}\text { Aluminum } \\
\text { hydroxide }\end{array}$ & $\begin{array}{l}\text { LC50 (16 days) } 430-3 \\
910 \mu \mathrm{g} / \mathrm{L} \\
\text { LC50 ( } 8 \text { days) } 22.4 \mathrm{mg} / \mathrm{L} \\
\text { LC50 ( } 4 \text { days) } 570-218 \\
644.1 \mu \mathrm{g} / \mathrm{L} \\
\text { LC50 (4 days) } 2.9 \mu \mathrm{mol} / \mathrm{L} \\
\text { LC50 (72 h) } 10-19.3 \\
\mathrm{mg} / \mathrm{L}\end{array}$ & $\begin{array}{l}\text { NOEC (60 days) } 88- \\
350 \mu \mathrm{g} / \mathrm{L} \\
\text { NOEC ( } 33 \text { days) } 71.5- \\
558.1 \mu \mathrm{g} / \mathrm{L} \\
\text { NOEC ( } 30 \text { days) } 57-88 \\
\mu \mathrm{g} / \mathrm{L} \\
\text { NOEC ( } 28 \text { days) } 4.7- \\
23.1 \mathrm{mg} / \mathrm{L} \\
\text { NOEC ( } 7 \text { days) } 25.1- \\
56476.6 \mu \mathrm{g} / \mathrm{L}\end{array}$ & $\begin{array}{l}\text { EC50 }(48 \mathrm{~h}) 1.5 \\
-2.56 \mathrm{mg} / \mathrm{L} \\
\text { LC50 }(4 \mathrm{days}) \\
22-30.6 \mathrm{mg} / \mathrm{L} \\
\text { LC50 }(48 \mathrm{~h}) 5.7 \\
-99600 \mu \mathrm{g} / \mathrm{L} \\
\text { NOEC }(4 \mathrm{days}) \\
22.6 \mathrm{mg} / \mathrm{L} \\
\text { NOEC }(48 \mathrm{~h}) 5- \\
671.2 \mu \mathrm{g} / \mathrm{L}\end{array}$ & $\begin{array}{l}\text { NOEC (42 days) } 232.6 \\
-453.8 \mu \mathrm{g} / \mathrm{L} \\
\text { NOEC ( } 30 \text { days) } 1.092 \\
-2.099 \mathrm{mg} / \mathrm{L} \\
\text { NOEC ( } 28 \text { days) } 53.1 \\
-4281.8 \mu \mathrm{g} / \mathrm{L} \\
\text { NOEC ( } 21 \text { days) } 76- \\
600 \mu \mathrm{g} / \mathrm{L} \\
\text { NOEC ( } 17 \text { days) } 962.5 \\
\mu \mathrm{g} / \mathrm{L}\end{array}$ & $\begin{array}{l}\text { EC50 (4 days) } 5.4- \\
570 \mu \mathrm{g} / \mathrm{L} \\
\text { EC50 }(72 \mathrm{~h}) 16.9-1 \\
799 \mu \mathrm{g} / \mathrm{L} \\
\operatorname{NOEC}(72 \mathrm{~h}) 4-600 \\
\mu \mathrm{g} / \mathrm{L} \\
\mathrm{LOEC}(72 \mathrm{~h}) 400-1 \\
000 \mu \mathrm{g} / \mathrm{L} \\
\text { EC10 }(72 \mathrm{~h}) 203- \\
3155000 \mathrm{ng} / \mathrm{L}\end{array}$ & No data \\
\hline
\end{tabular}


For invertebrates, the smallest effect of short-term toxicity is sodium bicarbonate, and the greatest one is chloride, oxide and aluminum hydroxide.

Analysis of the long-term toxicity of substances for aquatic invertebrates shows a significant variation in data and the difficulty in evaluating them. The most fully presented are the final values of EC10 / LC10 or NOEC for freshwater invertebrates. As one can see, the most dangerous compound is ammonium sulfate. At the same time, the lowest NOEC values, obtained for a different period (6-42 days) (Table 2), are characteristic of aluminum compounds. However, these data have a significant scatter, which complicates the objectivity of their comparison.

The toxicity of the tested compounds for algae and cyanobacteria is most fully characterized by EC50 values $(72 \mathrm{~h})$. As can be seen, toxicants such as aluminum compounds are the most dangerous for these organisms.

When analyzing the toxic effects of the tested inorganic compounds on aquatic microorganisms, the EC50 parameter was used $(3 \mathrm{~h})$. As can be seen (Table 2), the most dangerous compounds are sulfonic compounds and aluminum compounds, in particular sulfamic acid, basic aluminum chloride and aluminum sulfate. Based on scattered data, it can be assumed that magnesium and sodium chlorides, as well as ammonium sulfate, have the least toxic effect on aquatic microorganisms.

It can be said that in the short and long term, inorganic compounds based on aluminum, sulfamic acid and sodium bicarbonate are the most dangerous for aquatic living organisms. And the safest ones are magnesium and sodium chlorides. Incomplete data and their significant variability greatly complicate data processing.

Thus, despite the fragmentation and incompleteness of the available data, and their significant variability, including the parameters themselves and the conditions for obtaining them, for aquatic living organisms in the short and long term, the most dangerous are inorganic compounds based on aluminum, sulfamic acid and sodium bicarbonate, and the safest are magnesium and sodium chlorides.

\section{CONCLUSIONS}

It is advisable to analyze the effect of inorganic additives of foaming agents on the environment by studying the ecological, ecotoxicological and toxicological characteristics of inorganic salts, which are used to improve the extinguishing properties of foaming agents, taking into account their effect on living organisms and the environment.

A comparative analysis of the environmental hazards of the tested inorganic additives of foaming agents showed that the most dangerous for the environment are aluminum compounds and sulfamic acid, and the safest are magnesium and sodium chlorides.

Despite the fragmentation and incompleteness of the available data, and their significant variability, including the parameters themselves and the conditions for obtaining them, for aquatic living organisms in the short and long term, the most dangerous are inorganic compounds based on aluminum, sulfamic acid and sodium bicarbonate, and the safest are magnesium and sodium chlorides.

\section{REFERENCES}

1. Abramov YA, Basmanov OE, Salamov J, Mikhayluk AA Model of thermal effect of fire within a dike on the oil tank. Naukovyi Visnyk Natsionalnoho Hirnychoho Universytetu. 2018 2:95-100 DOI: $10.29202 /$ nvngu/2018-2/12.

2. The race to decipher how climate change influenced Australia's record fires. Available at: https://www.nature.com/articles/d41586-02000173-7.

3. World Fire Statistics. Available at: https://www.ctif.org/world-fire-statistics.

4. Dubinin D, Korytchenko K, Lisnyak A, Hrytsyna I, Trigub V. Numerical simulation of the creation of a fire fighting barrier using an explosion of a combustible charge. EasternEuropean Journal of Enterprise Technologies. 2017 6(10-90):11-16 DOI: $10.15587 / 1729-4061.2017 .114504$

5. Andronov V, Pospelov B, Rybka E, Skliarov S. Examining the learning fire detectors under real conditions of application. EasternEuropean Journal of Enterprise Technologies. 2017 3(9-87):53-59 DOI: $10.15587 / 1729-4061.2017 .101985$.

6. Mygalenko $K$, Nuyanzin $V$, Zemlianskyi A, Dominik A, Pozdieiev S. Development of the technique for restricting the propagation of fire in natural peat ecosystems. EasternEuropean Journal of Enterprise Technologies. 2018 1(10-91):31-37 DOI: $10.15587 / 1729-4061.2018 .121727$.

7. Kostenko V, Kostenko T, Zemlianskiy O, Maiboroda A, Kutsenko S. Automatization of individual anti-thermal protection of rescuers in the 
initial period of fire suppression. EasternEuropean Journal of Enterprise Technologies. 2017 5(10-89): 4-11 DOI: $10.15587 / 1729-4061.2017 .109484$.

8. Vasiliev MI, Movchan IO, Koval OM. Diminishing of ecological risk via optimization of fireextinguishing system projects in timber-yards. Naukovyi Visnyk Natsionalnoho Hirnychoho Universytetu. 2014 5:106-113.

9. Holemann H. Environmental Problems Caused by Fires and Fire-Fighting Agents. Available at: http://www.iafss.org/publications/fss/4/61/view.

10. Loboichenko V, Strelets $V$, Gurbanova $M$, Morozov A, Kovalov P, Shevchenko R, Kovalova T and Ponomarenko R. Review of the Environmental Characteristics of Fire Extinguishing Substances of Different Composition used for Fires Extinguishing of Various Classes. Journal of Engineering and Applied Sciences. 2019 14: 5925-5941.

11. Tureková I \& Balog K. The Environmental Impacts of Fire-Fighting Foams. Research Papers Faculty of Materials Science and Technology Slovak University of Technology. 2011 18(29):111-120 doi: 10.2478/v10186-010-0033-z.

12. Dubinin D, Korytchenko K, Lisnyak A, Hrytsyna $\mathrm{I}$, Trigub $\mathrm{V}$. Improving the installation for fire extinguishing with inelydispersed water. EasternEuropean Journal of Enterprise Technologies. 2018 2(10-92):38-43 DOI: $10.15587 / 1729-4061.2018 .127865$.

13. Loboichenko $V$, Leonova N, Strelets $V$, Morozov A, Shevchenko R, Kovalov P, Ponomarenko R and Kovalova $T$ Comparative Analysis of the Influence of Various Dry Powder Fire Extinguishing Compositions on the Aquatic Environment. Water and Energy International. 2019 62/RNI(7):63-68.

14. Sharovarnikov AF, Sharovarnikov SA. Penoobrazovateli i peny dlya tusheniya pozharov. Sostav, svoystva, primeneniye. M: Pozhnauka. 2005335 p (in Russian).

15. Vasyukov A, Loboichenko V, Bushtec S. Identification of bottled natural waters by using direct conductometry. Ecology, Environment and Conservation. 201622 (3):1171-1176.

16. Loboichenko $\mathrm{V}$, Andronov $\mathrm{V}$, Strelec $\mathrm{V}$. Evaluation of the metrological characteristics of natural and treated waters with stable salt composition identification method. Indian Journal of Environmental Protection. 2018 38(9):724-732.

17. Osnovy analiticheskoy khimii. V 2 kn. Kn. 2. Metody khimicheskogo analiza. Ucheb. dlya vuzov / Zolotov YuA (Ed.). 2004 M: «Vysshaya shkola», 503 p. (in Russian)

18. Loboichenko VM, Tishakova TS, Vasyukov AE. Application of direct coulometry for rapid assessment of water quality in Krasno-Oskol Reservoir (Kharkiv Region, Ukraine). Der Pharma Chemica. 20168 (19):27-34.

19. Loboichenko VM, Vasyukov AE, Tishakova TS. Investigations of Mineralization of Water Bodies on the Example of River Waters of Ukraine. Asian Journal of Water, Environment and Pollution. 2017 14 (4):37-41 DOI: 10.3233/AJW-170035.

20. Kawahara T, Hatae Sh, Kanyama T, Ishizaki $Y$, Uezu K. Development of Eco-Friendly Soap-Based Firefighting Foam for Forest Fire. Environ. Control Biol. 2016 54(1):75-78 DOI: 10.2525/ecb.54.75/.

21. Kawano $T$, Otsuka K, Kadono T, Inokuchi R, Ishizaki $Y$, Dewancker $B$ and Uezu K. EcoToxicological Evaluation of Fire-Fighting Foams in Small-Sized Aquatic and Semi-Aquatic Biotopes. Advanced Materials Research. 2014 875-877:699707.

22. Levterov AM, Levterov AA. Thermodynamic properties of fatty acid esters in some biodiesel fuels. Functional Materials. 2018 25(2):308-312 DOI: $10.15407 / \mathrm{fm} 25.02 .308$.

23. Chirkina M, Saveliev D, Pitak O. Possibility of using eco-friendly foams for fire suppression. Problems of fire safety. $201742: 176-180$ (in Russian)

24. Korolchenko DA, Volkov AA. Extinguishing of flammable liquids by film forming foaming agents. Pozharovzryvobezopasnost/Fire and Explosion Safety. 2017 26(8):45-55. https://doi.org/10.18322/PVB.2017.26.08.45-55 (in Russian)

25. Seow J. Department of Environment and Conservation Western Australia, 2013, Fire Fighting Foam with perfluorochemical environmental review. Available at: http://www.hemmingfire.com/news/fullstory.php/ aid/1748/The final definitive version of 91Fire Fighting Foams with Perfluorochemicals 96 Envi ronmental Review 92, by Dr Jimmy Seow, Man ager, Pollution Response Unit, Department of $E$ nvironment and Conservation Western Australia. $\underline{\text { html. }}$

26. Goto K, Takaichi $\mathrm{H}$ and Kawano T. Learning from the Eco-Toxicology of Fire-Fighting Foams in Aquatic Organisms: Altered Eco-Toxicity of Sodium Alkyl Sulfonates on Green Paramecia and Medaka 
Fish Maintained in Different Waters. J. Disaster Res. 2015 10(4):604-612.

27. Globally harmonized system of classification and labelling of chemicals (GHS). United Nations, New York, USA. Available at: http://www.unece.org/ru/trans/danger/publi/ghs/g hs_rev07/07files_e0.html.

28. Database of the European Chemicals Agency. Available at: https://echa.europa.eu/home.

29. GOST R 53857-2010: Classification of chemicals for environmental hazards. General principles. 2011.

30. Dadashov I, Loboichenko $\mathrm{V}$ and Kireev $\mathrm{A}$. Analysis of the ecological characteristics of environment friendly fire fighting chemicals used in extinguishing oil products. Pollution Research. 2018 37:63-77.

31. Patent RU 2403935. Foaming composition of heat-resistant foam to extinguish fires at subzero temperatures. Taysumov HA. 2010. Bul. № 32. (in Russian)

32. Patent RU 2457879. Foaming composition of heat-resistant foam for the fuel tank safety cover. Taysumov HA. 2012. Bul. № 22. (in Russian)
33. Patent RU 2418611C1. Fire extinguishing composition for fire fighting. Garavin VYu, Tretyakov AV. 2011. Bul. № 14. (in Russian)

34. Patent RU 2290240. Fire extinguishing composition. Dushkin AL., Karpyshev AV. 2006. Bul. № 36. (in Russian)

35. Patent SU 865303. The composition of the foaming agent to extinguish fires. Kazakov MV., Bilkun DG., Peshkov VV, Puzako M.V. 1981. Bul. № 35. (in Russian)

36. Patent US 3554912. Basic Aluminum Salt Fire Extinguishing compositions. EP Moore Jr. Patented Jan. 12, 1971.

37. Patent RU 2465028. Environmentally friendly foaming composition of heat-resistant foam. Taysumov HA. 2010. Bul. № 32. (in Russian)

38. Patent RU 2452544. Foaming composition of heat-resistant hop-based foam. Taysumov HA. 2012. Bul. № 16. (in Russian)

39. Patent RU 2328325. Concentrated stabilizer of heat-resistant foam to extinguish fires. Taysumov HA. 2008. Bul. № 19. (in Russian)

40. GB 748931A. United Kingdom. Improvements in or relating to the production of foam for firefighting purposes. John Kerr and Co Manchester Itd. Published 1956-05-16. 
